\title{
PRESERVATION OF LOG-CONCAVITY ON SUMMATION
}

\author{
Oliver JOHnson $^{1,2}$ And Christina GoldsChmidt ${ }^{1,3}$
}

\begin{abstract}
We extend Hoggar's theorem that the sum of two independent discrete-valued log-concave random variables is itself log-concave. We introduce conditions under which the result still holds for dependent variables. We argue that these conditions are natural by giving some applications. Firstly, we use our main theorem to give simple proofs of the log-concavity of the Stirling numbers of the second kind and of the Eulerian numbers. Secondly, we prove results concerning the log-concavity of the sum of independent (not necessarily log-concave) random variables.
\end{abstract}

Mathematics Subject Classification. 60E15, 60C05, 11B75.

Received April 15, 2005.

\section{INTRODUCTION AND MAIN THEOREM}

The property of log-concavity $(\mathrm{LC})$ for non-negative sequences is defined as follows.

Definition 1.1. A sequence $(u(i), i \geq 0)$ is $\log$-concave if, for all $i \geq 1$,

$$
u(i)^{2} \geq u(i-1) u(i+1) .
$$

Equation (1) is sometimes referred to the quadratic Newton inequality (see Niculescu [13]), and plays a role in considering when polynomials with real coefficients have real roots (see Sect. 3). Applications of log-concavity arise in combinatorics, algebra, geometry and computer science, as reviewed by Stanley [17] and Brenti $[4,6]$. In probability and statistics, it is related to the notion of negative association of random variables (see Joag-Dev and Proschan [10]).

Definition 1.2. A random variable $V$ taking values in $\mathbb{Z}_{+}$is log-concave if its probability mass function $p_{V}(i)=\mathbb{P}(V=i)$ forms a log-concave sequence. That is, $V$ is log-concave if for all $i \geq 1$,

$$
p_{V}(i)^{2} \geq p_{V}(i-1) p_{V}(i+1)
$$

\section{Example 1.3.}

(1) We write $\operatorname{Geom}(p)$ for the geometric distribution with mass function $p_{X}(i)=(1-p) p^{i}$, for $i \in \mathbb{Z}_{+}$. For each $p$, these random variables represent the "edge case" of the log-concavity property, in that equation (2) becomes an identity for all $i \geq 1$.

\footnotetext{
Keywords and phrases. Log-concavity, convolution, dependent random variables, Stirling numbers, Eulerian numbers.

1 Statistical Laboratory, Centre for Mathematical Sciences, University of Cambridge, Wilberforce Rd, Cambridge, CB3 0WB, UK.

2 Christ's College, Cambridge; otj1000@cam.ac.uk

3 Pembroke College, Cambridge; C.Goldschmidt@statslab.cam.ac.uk
} 
(2) The $\operatorname{Poisson}(\lambda)$ distribution is log-concave for any $\lambda \geq 0$.

(3) Any Bernoulli random variable (that is, only taking values in $\{0,1\}$ ) is log-concave. Further, any binomial distribution is log-concave. In fact, any random variable $S=\sum_{i=1}^{n} X_{i}$, where $X_{i}$ are independent (not necessarily identical) Bernoulli variables, is log-concave.

This last fact is a corollary of the following theorem, first proved by Hoggar [9].

Theorem 1.4. If $V$ and $W$ are independent log-concave random variables, then their sum $V+W$ is also log-concave. Equivalently, the convolution of any two log-concave sequences is log-concave.

A similar result was proved by Davenport and Pólya [7], under the condition that the probability mass functions of $V$ and $W$, rescaled by binomial coefficients, are log-concave.

Notice that the sum of two independent and identically distributed geometric random variables (both on the edge case) is a negative binomial distribution (still log-concave, but no longer the edge case), suggesting room for improvement. In Section 2, we prove the following main theorem, an extension of Theorem 1.4 to the case of dependent random variables.

Theorem 1.5. If $V$ is a log-concave random variable and random variables $V$, $W$ satisfy Condition 1 below then the sum $V+W$ is also log-concave.

We give two distinct applications of Theorem 1.5 in the remaining sections of the paper. Firstly, we consider the important property of log-concavity of certain combinatorial sequences. It is often desirable to show that such sequences are unimodal (a sequence $c(\cdot)$ is unimodal if there exists an index $j$ such that $c(i) \leq c(i+1)$ for $i \leq j-1$ and $c(i) \geq c(i+1)$ for $i \geq j)$. Log-concavity is easily shown to imply unimodality and, since unimodality is not preserved on convolution, log-concavity is often easier to prove. The motivation for Hoggar's paper [9] came from the coefficient sequences of chromatic polynomials. For a graph $G$, the chromatic polynomial $\pi_{G}(z)=\sum_{i} c(i) z^{i}$ gives the number of ways to colour $G$ using exactly $z$ colours. Read [14] conjectured that $(|c(i)|, i \geq 0)$ is a unimodal sequence and Welsh [21] conjectured, more strongly, that it is log-concave. Hoggar proved Theorem 1.4 in a partial attempt to resolve this conjecture. It remains unproven, but progress towards it is reviewed in Brenti [5].

In Section 3, we show how a version of Theorem 1.5 (adapted to apply to sequences rather than just probability mass functions) can be used to prove the log-concavity of certain combinatorial sequences. In particular, in Theorem 3.2 we use it to give a simple proof of the log-concavity of the Stirling numbers of the second kind (Condition 1 is natural here and straightforward to check). In Theorem 3.3, we prove log-concavity of the Eulerian numbers, even though our Condition 1 fails in this case, by a simple adaptation of our methods.

The second application comes in Section 4, where for fixed log-concave $V$ we consider the set of independent random variables $W$ such that $V+W$ is log-concave. This means that we weaken one of Hoggar's assumptions, since we no longer require $W$ to be log-concave. We prove Lemma 4.3, which gives simple conditions under which $X+\operatorname{Geom}(p)$ is log-concave, for independent $X$ and Geom $(p)$. Further, we extend the arguments of Theorem 1.5 to prove Theorem 4.4, which states that if $X+\operatorname{Geom}\left(p_{1}\right)$ is $\log$-concave, then so is $X+\operatorname{Geom}\left(p_{2}\right)$ for $p_{2} \geq p_{1}$. (This result is not unexpected, but it cannot be stated as a simple corollary of Hoggar's Theorem). This result is relevant to the field of reliability theory, where we may wish to establish the log-concavity (and hence bounds on the hazard rate) of the total lifetime of a collection of components, where some of the components have a geometric lifetime with unknown (but bounded below) parameter $p$. This restriction on the geometric parameter is very natural if we consider trying to infer $p$ from a collection of lifetimes while the trials are ongoing and some components "haven't failed yet".

Log-concavity is a property much used in econometrics, as described in the survey by Bergstrom and Bagnoli [1]. A previous attempt to generalise Hoggar's theorem in this context, by Brais, Martimort and Rochet [2], led to the statement of the incorrect result that for independent $V$ and $W$ if either of $V$ or $W$ is log-concave then their sum must be (see the discussion in Miravete [12]). It is clear, however, that extensions of Hoggar's theorem may have applications here.

A version of Theorem 1.5 where $W$ must be a Bernoulli random variable is proved in a different context by Sagan [15] (Th. 1). Our conditions reduce to his in this special case. Wang [18] proves that linear transformations 
based on binomial coefficients preserve log-concavity (see also [20]). An anonymous referee brought it to our attention that, at about the same time as the first draft of this paper, Wang and Yeh had independently submitted a paper [19] which contains some similar technical results. In particular, our Lemma 2.4 corresponds to their Lemma 2.1 and our Condition 1 corresponds to their Lemma 2.3.

\section{Conditions and Proof of Theorem 1.5}

We prove a generalized version of Theorem 1.5, which establishes both results about the log-concavity of the sum of dependent random variables and results about the log-concavity of a extended version of convolutions of sequences.

In general, given sequences $p_{V}(v)$ and $p_{V+W}(x)$, we will suppose that there exists a two-dimensional array of coefficients $p_{W \mid V}(w \mid v)$ such that

$$
p_{V+W}(x)=\sum p_{V}(v) p_{W \mid V}(x-v \mid v) .
$$

In essence, the $p_{W \mid V}$ act like conditional probabilities, but with no need for the sequences to sum to 1 .

We give here an example of a pair of dependent geometric random variables whose sum is also geometric, motivating Condition 1 below, under which we prove our extension of Hoggar's theorem.

Example 2.1. For some $p \in(0,1)$ and $\alpha \in(0,1)$, define the joint distribution of $V$ and $W$ by

$$
\mathbb{P}(V=i, W=j)=\left(\begin{array}{c}
i+j \\
i
\end{array}\right)(1-p) p^{i+j} \alpha^{i}(1-\alpha)^{j}, \text { for } i, j \geq 0 .
$$

Using the identities $\sum_{i=0}^{k}\left(\begin{array}{c}k \\ i\end{array}\right) \alpha^{i}(1-\alpha)^{k-i}=1$ and $\sum_{j=0}^{\infty}\left(\begin{array}{c}i+j \\ i\end{array}\right) t^{j}=(1-t)^{-i-1}$ (for $\left.0 \leq t<1\right)$, we deduce that $V+W$ is $\operatorname{Geom}(p), V$ is $\operatorname{Geom}(\alpha p /(\alpha p+(1-p))$ and $W$ is $\operatorname{Geom}(p /((1-\alpha) p+(1-p)))$. The conditional probabilities are negative binomial with

$$
p_{W \mid V}(j \mid i)=\mathbb{P}(W=j \mid V=i)=\left(\begin{array}{c}
i+j \\
i
\end{array}\right)(1-p+\alpha p)^{i+1}(p(1-\alpha))^{j}
$$

Definition 2.2. Given coefficients $p_{W \mid V}$ and fixed $i$, define

$$
a_{r, s}^{(i)}=p_{W \mid V}(i-r \mid r) p_{W \mid V}(i-s \mid s)-p_{W \mid V}(i-r-1 \mid r) p_{W \mid V}(i-s+1 \mid s) .
$$

Condition 1. For the quantities $a_{r, s}^{(i)}$ defined above, we require that for all $0 \leq t \leq m \leq i$,

$$
\text { (a) } \sum_{k=-t}^{t} a_{m+k, m-k}^{(i)} \geq 0 \text { and (b) } \sum_{k=-t-1}^{t} a_{m+k+1, m-k}^{(i)} \geq 0 \text {. }
$$

Remark 2.3. Note that Condition 1 holds in Example 2.1. The key is to observe that, in this case, for any given $i, a_{r, s}^{(i)}$ is proportional to $\left(\begin{array}{l}i \\ r\end{array}\right)\left(\begin{array}{l}i \\ s\end{array}\right)-\left(\begin{array}{c}i-1 \\ r\end{array}\right)\left(\begin{array}{c}i+1 \\ s\end{array}\right)$. This means that for Condition $1(\mathrm{a})$, as $t$ increases, the increment term $a_{m+t, m-t}^{(i)}+a_{m-t, m+t}^{(i)}$ is proportional to $2\left(\begin{array}{c}i \\ m+t\end{array}\right)\left(\begin{array}{c}i \\ m-t\end{array}\right)-\left(\begin{array}{c}i-1 \\ m+t\end{array}\right)\left(\begin{array}{c}i+1 \\ m-t\end{array}\right)-\left(\begin{array}{c}i+1 \\ m+t\end{array}\right)\left(\begin{array}{c}i-1 \\ m-t\end{array}\right)$, which is positive for $t \leq T$ and negative for $t>T$ (for some value $T$ ). Hence, the partial sums, $\sum_{k=-t}^{t} a_{m+k, m-k}^{(i)}$, form a sequence which increases for $t \leq T$ and decreases thereafter. Using the identity $\sum_{j}\left(\begin{array}{c}a \\ j\end{array}\right)\left(\begin{array}{c}b \\ r-j\end{array}\right)=\left(\begin{array}{c}a+b \\ r\end{array}\right)$, we see that $\sum_{k=-m}^{m} a_{m+k, m-k}^{(i)}=0$ and so the sequence of partial sums must be non-negative for any $t$. A similar argument holds for Condition 1(b).

In order to prove Theorem 1.5 we require a technical lemma. 
Lemma 2.4. Fix $l \geq m$ and suppose that $\left(c_{j}\right)$ is a sequence such that $C_{n}:=\sum_{j=0}^{n} c_{j} \geq 0$ for all $0 \leq n \leq m$. For any log-concave sequence $p$, and for any $0 \leq i \leq m$,

$$
\sum_{j=0}^{i} p(l+j) p(m-j) c_{j} \geq 0
$$

(This result is obvious if each $c_{j} \geq 0$, but the condition $C_{n} \geq 0$ for all $0 \leq n \leq m$ is evidently weaker.)

Proof. We apply Abel's summation formula ("summation by parts") to obtain that

$$
\begin{aligned}
& \sum_{j=0}^{i} p(l+j) p(m-j) c_{j}=\sum_{j=0}^{i} p(l+j) p(m-j)\left(C_{j}-C_{j-1}\right) \\
& =\sum_{j=0}^{i}(p(l+j) p(m-j)-p(l+j+1) p(m-j-1)) C_{j}+C_{i} p(l+i+1) p(m-i-1),
\end{aligned}
$$

where, by convention, $C_{-1}=0$. The log-concavity of $p$ implies that $p(l+j) p(m-j) \geq p(l+j+1) p(m-j-1)$ for $j \geq 0$ and so, since each $C_{j} \geq 0$, the result follows.

Proof of Theorem 1.5. For any $i$, the sequence $p_{V+W}$ defined by (4) satisfies

$$
\begin{aligned}
p_{V+W}(i)^{2}-p_{V+W}(i-1) p_{V+W}(i+1) \\
\quad=\sum_{j=0}^{i} \sum_{k=0}^{i+1} p_{V}(j) p_{V}(k)\left\{p_{W \mid V}(i-j \mid j) p_{W \mid V}(i-k \mid k)-p_{W \mid V}(i-j-1 \mid j) p_{W \mid V}(i-k+1 \mid k)\right\} \\
\quad=\sum_{j=0}^{i} \sum_{k=0}^{i+1} p_{V}(j) p_{V}(k) a_{j, k}^{(i)} .
\end{aligned}
$$

For simplicity, we decompose the above sum into three regions: (a) $\{j \geq k\}$ (b) $\{j=k-1\}$ (c) $\{j \leq k-2\}$. We relabel the third region, using the new co-ordinates $(J, K)=(k-1, j+1)$, which transforms it into $\{J \geq K\}$. This enables us to rewrite Equation (5) as

$$
\begin{aligned}
& \sum_{j=0}^{i} p_{V}(j) p_{V}(j+1) a_{j, j+1}^{(i)}+\sum_{i \geq j \geq k}\left(p_{V}(j) p_{V}(k) a_{j, k}^{(i)}+p_{V}(j+1) p_{V}(k-1) a_{k-1, j+1}^{(i)}\right) \\
& =\sum_{m=0}^{i}\left\{\sum_{k=0}^{m}\left[p_{V}(m+k) p_{V}(m-k) a_{m+k, m-k}^{(i)}+p_{V}(m+k+1) p_{V}(m-k-1) a_{m-k-1, m+k+1}^{(i)}\right]\right. \\
& +p_{V}(m) p_{V}(m+1) a_{m, m+1}^{(i)} \\
& \left.+\sum_{k=1}^{m+1}\left[p_{V}(m+k) p_{V}(m-k+1) a_{m+k, m-k+1}^{(i)}+p_{V}(m+k+1) p_{V}(m-k) a_{m-k, m+k+1}^{(i)}\right]\right\} .
\end{aligned}
$$

Here the second term in curly brackets corresponds to the first term in equation (6). The first and third terms correspond to the second term in equation (6), split according to whether $r=j+k$ is even or odd. If $r$ is even 
then $m=r / 2$; if $r$ is odd then $m=(r-1) / 2$. Consider now the first term in (7):

$$
\begin{aligned}
& \sum_{k=0}^{m}\left[p_{V}(m+k) p_{V}(m-k) a_{m+k, m-k}^{(i)}+p_{V}(m+k+1) p_{V}(m-k-1) a_{m-k-1, m+k+1}^{(i)}\right] \\
& =\sum_{k=0}^{m} p_{V}(m+k) p_{V}(m-k) a_{m+k, m-k}^{(i)}+\sum_{k=0}^{m-1} p_{V}(m+k+1) p_{V}(m-k-1) a_{m-k-1, m+k+1}^{(i)} \\
& =p_{V}(m)^{2} a_{m, m}^{(i)}+\sum_{k=1}^{m} p_{V}(m+k) p_{V}(m-k)\left\{a_{m+k, m-k}^{(i)}+a_{m-k, m+k}^{(i)}\right\} \\
& =\sum_{k=0}^{m} p_{V}(m+k) p_{V}(m-k) c_{k},
\end{aligned}
$$

where $c_{0}=a_{m, m}^{(i)}$ and $c_{k}=a_{m+k, m-k}^{(i)}+a_{m-k, m+k}^{(i)}$ for $1 \leq k \leq m$. Then Condition 1 (a) tells us that $\sum_{k=0}^{t} c_{k} \geq 0$ for all $0 \leq t \leq m$ and so, by Lemma 2.4 with $l=m, i=m$, equation (8) is positive. In the same way, we can show that sum of the second and third terms in (7) equals

$$
\sum_{k=0}^{m} p_{V}(m+k+1) p_{V}(m-k) d_{k}
$$

where $d_{k}=a_{m+k+1, m-k}^{(i)}+a_{m-k, m+k+1}^{(i)}$ for $0 \leq k \leq m$. Then Condition 1(b) tells us that $\sum_{k=0}^{t} d_{k} \geq 0$ for all $0 \leq t \leq m$ and so, by Lemma 2.4 with $l=m+1, i=m$, equation (9) is positive.

Hence, $p_{V+W}(i)^{2}-p_{V+W}(i-1) p_{V+W}(i+1) \geq 0$ for all $i \geq 0$.

We now identify which properties of independent random variables allow Hoggar's result, Theorem 1.4, to be proved. In particular, Condition 1 holds under Hoggar's assumptions, so Theorem 1.5 implies Theorem 1.4.

Remark 2.5. For independent random variables $V$ and $W$, the $a_{j, k}^{(i)}$ have the following properties: (a) for all $j, a_{j, j+1}^{(i)} \equiv 0$ (b) for $j \geq k, a_{k-1, j+1}^{(i)}=-a_{j, k}^{(i)}$ (c) if $W$ is log-concave then $a_{j, k}^{(i)} \geq 0$ for $j \geq k$.

Hence, if we fix $i$ and define $c_{j}$ and $d_{j}$ as in the proof of Theorem 1.5, for $V$ and $W$ independent and log-concave we can write:

$$
\begin{aligned}
\sum_{j=0}^{t} c_{j} & =a_{m, m}^{(i)}+\sum_{j=1}^{t} a_{m+j, m-j}^{(i)}-\sum_{j=1}^{t} a_{m+j-1, m-j+1}^{(i)} \\
& =a_{m, m}^{(i)}+\sum_{j=1}^{t} a_{m+j, m-j}^{(i)}-\sum_{j=0}^{t-1} a_{m+j, m-j}^{(i)}=a_{m+t, m-t}^{(i)} \geq 0 .
\end{aligned}
$$

Similarly:

$$
\begin{aligned}
\sum_{j=0}^{t} d_{j} & =\sum_{j=0}^{t} a_{m+1+j, m-j}^{(i)}-\sum_{j=0}^{t} a_{m+j, m-j+1}^{(i)} \\
& =a_{m+1+t, m-t}^{(i)}-a_{m, m+1}^{(i)}=a_{m+1+t, m-t}^{(i)} \geq 0 .
\end{aligned}
$$

Thus, Condition 1 holds for independent and log-concave $V$ and $W$. 


\section{LOG-CONCAVITY OF COMBINATORIAL SEQUENCES}

Many common combinatorial sequences, such as the binomial coefficients $\left(\left(\begin{array}{l}n \\ k\end{array}\right), 0 \leq k \leq n\right)$, are log-concave in $k$. A key result for proving the log-concavity of combinatorial sequences is the following.

Lemma 3.1. If all the roots of the generating function $p(x)=c(0) x^{n}+c(1) x^{n-1}+c(2) x^{n-2}+\cdots+c(n)$ are real and negative, then its coefficients $c(k)$ form a log-concave sequence.

The proof can be regarded as an application of Theorem 1.4. See Niculescu [13] for a review of this and similar properties.

Section 4.5 of Wilf [22] contains proofs of log-concavity for the binomial coefficients and the Stirling numbers of the first and second kinds. He shows that the generating function of each sequence has real and negative roots, and then appeals to Lemma 3.1 to deduce log-concavity of the sequence.

For the first two examples he considers, this is relatively straightforward. That is, in Corollary 4.5.1 he uses the fact that the binomial coefficients $\left(\begin{array}{l}n \\ k\end{array}\right)$ have generating function $(1+x)^{n}$, and in Corollary 4.5.2 he uses the fact that the Stirling numbers of the first kind have generating function $(x+1)(x+2) \ldots(x+n-1)$.

However, the fact that these generating functions factorise into products of independent terms indicate that both these examples can equally well be dealt with via Hoggar's Theorem. As remarked in Example 1.3.3, the binomial distribution is log-concave. Further, the fact that the Stirling numbers of the first kind satisfy the recurrence relation $s(n+1, k)=s(n, k-1)+n s(n, k)$ means that we can write $s(n, k)=n ! \mathbb{P}\left(X_{1}+\ldots+X_{n}=k\right)$ (the normalization is irrelevant here), where $X_{i}$ are independent with $\mathbb{P}\left(X_{i}=0\right)=(i-1) / i$ and $\mathbb{P}\left(X_{i}=1\right)=1 / i$, so the result derived by Wilf is simply another case of Example 1.3.3.

Indeed, Hoggar's theorem can prove log-concavity in cases where the method described by Wilf fails. For fixed $n$, let $b(n, k)$ be the number of permutations of $n$ objects with exactly $k$ inversions (that is, the permutation $\sigma$ has exactly $k$ pairs $i<j$ such that $\sigma(i)>\sigma(j))$. Equation (4.5.7) of [22] shows that the generating function of $b(n, k)$ takes the form

$$
p(x)=\prod_{i=1}^{n-1}\left(1+x+\ldots+x^{i}\right) .
$$

This means that the sequence $b(n, k)$ can be expressed as the convolution of independent log-concave sequences of the form $(1,1, \ldots, 1)$, and so is $\log$-concave. However, for $n \geq 3$, the generating function $p(x)$ has imaginary roots, so Lemma 3.1 does not apply.

The Stirling numbers of the second kind provide an example where Wilf's proof is relatively complicated and where Hoggar's result does not apply. However, our Theorem 1.5 gives a simple proof in this case, using only the property that $S(n, k)=S(n-1, k-1)+k S(n-1, k)$. In fact, this property is used by Sagan [15] in his proof of the same result; however, his techniques only apply in the case where $W$ is a $\{0,1\}$-valued random variable, whereas our Theorem 1.5 will prove log-concavity of a more general family of combinatorial sequences.

Theorem 3.2. For given $n$, the Stirling numbers of the second kind, $S(n, k)$ form a log-concave sequence in $k$.

Proof. In the notation of equation $(3)$, if $p_{V}(i)=S(n-1, i)$ then $p_{V+W}(i)=S(n, i)$, where for each $j \leq n-1$,

$$
p_{W \mid V}(x \mid j)= \begin{cases}j & \text { if } x=0 \\ 1 & \text { if } x=1 \\ 0 & \text { otherwise }\end{cases}
$$

(so that $V$ and $W$ are not "independent"). Given $i$, for $j \geq k$, the only non-zero $a_{j, k}^{(i)}$ are

\begin{tabular}{c||c|c|c}
$a_{j, k}^{(i)}$ & $k=i-1$ & $k=i$ & $k=i+1$ \\
\hline \hline$j=i-2$ & 0 & -1 & $-(i+1)$ \\
$j=i-1$ & 1 & $i-(i-1)$ & $-(i+1)(i-1)$ \\
$j=i$ & $i$ & $i^{2}$ & 0
\end{tabular}


Hence, the only non-zero sequences of partial sums in Condition 1(a) are the case $m=i-1$, where $\left(\sum_{k=-t}^{t} a_{m+k, m-k}^{(i)}, t \geq 0\right)$ is $(1,0,0, \ldots)$, and the case $m=i$, where $\left(\sum_{k=-t}^{t} a_{m+k, m-k}^{(i)}, t \geq 0\right)$ is $\left(i^{2}, 1,1, \ldots\right)$. In Condition 1(b), the only non-zero sequence of partial sums occurs for $m=i-1$, where $\left(\sum_{k=-t-1}^{t} a_{m+k+1, m-k}^{(i)}, t \geq 0\right)$ is $(i, i+1,0,0, \ldots)$. These are all positive, so Condition 1 holds, as required.

This result was first proved by Lieb [11]; see Wang and Yeh [20] for an alternative approach.

The proof of Theorem 3.2 can also be used to show that the $q$-Stirling numbers of the second kind, $\left(S^{(q)}(n, k), 0 \leq k \leq n\right)$, form a log-concave sequence for $0 \leq q \leq 1$ (see Sagan [16] for the definition of the $q$-Stirling numbers and a proof of this fact for all $q \geq 0$ ).

Indeed, our methods can sometimes be adapted to prove log-concavity of combinatorial sequences even when Condition 1 and Theorem 1 of [15] fail. For example, consider the Eulerian numbers $E(n, k)$, the number of permutations of $n$ objects with exactly $k$ ascents (that is, the permutation $\sigma$ has exactly $k$ places $j$ such that $\sigma(j)<\sigma(j+1)$ ). We prove the following well-known result (see, for example, Gasharov [8] or Bóna and Ehrenborg [3] for alternative proofs).

Theorem 3.3. For given $n$, the Eulerian numbers $E(n, k)$ form a log-concave sequence in $k$.

Proof. The recurrence relation $E(n, k)=(k+1) E(n-1, k)+(n-k) E(n-1, k-1)$, implies that

$$
p_{W \mid V}(x \mid j)= \begin{cases}j+1 & \text { if } x=0 \\ n-j & \text { if } x=1 \\ 0 & \text { otherwise. }\end{cases}
$$

Given $i$, for $j \geq k$, the only non-zero $a_{j, k}^{(i)}$ are

\begin{tabular}{c||c|c|c}
$a_{j, k}^{(i)}$ & $k=i-1$ & $k=i$ & $k=i+1$ \\
\hline \hline$j=i-2$ & 0 & $-(n-i)(n-i+2)$ & $-(i+2)(n-i+2)$ \\
$j=i-1$ & $(n-i+1)^{2}$ & $n+1$ & $-i(i+2)$ \\
$j=i$ & $(i+1)(n-i+1)$ & $(i+1)^{2}$ & 0
\end{tabular}

For $m=i-1$, Condition 1(b) fails. However, since we are attempting to show that $\sum_{j, k} a_{j, k}^{(i)} p_{V}(j) p_{V}(k)$ is positive, it will be sufficient to show that this sum minus a positive quantity is positive. If we define

$$
\sum_{j=0}^{i} \sum_{k=0}^{i+1} \widetilde{a}_{j, k}^{(i)} p_{V}(j) p_{V}(k)=\sum_{j=0}^{i} \sum_{k=0}^{i+1} a_{j, k}^{(i)} p_{V}(j) p_{V}(k)-\left(p_{V}(i-1)-p_{V}(i)\right)^{2},
$$

then the new coefficients are

\begin{tabular}{c||c|c|c}
$\widetilde{a}_{j, k}^{(i)}$ & $k=i-1$ & $k=i$ & $k=i+1$ \\
\hline \hline$j=i-2$ & 0 & $-(n-i)(n-i+2)$ & $-(i+2)(n-i+2)$ \\
$j=i-1$ & $(n-i+1)^{2}-1$ & $(n+1)+1$ & $-i(i+2)$ \\
$j=i$ & $(i+1)(n-i+1)+1$ & $(i+1)^{2}-1$ & 0
\end{tabular}

These new coefficients $\widetilde{a}_{j, k}^{(i)}$ do satisfy Condition 1 , so we deduce that the original difference $p_{V+W}(i)^{2}-p_{V+W}(i-$ 1) $p_{V+W}(i+1)$ is positive. 


\section{Development for the independent CASE}

We now use a version of Theorem 1.5 to give results about when the sum of independent random variables $V$ and $W$ is log-concave, even though $V$ and $W$ need not be.

To give a concrete example of an independent pair of random variables $V$ and $W$ such that $V+W \in L C$ but $W \notin L C$, let $W$ have probability mass function $p_{W}(0)=5 / 8, p_{W}(1)=1 / 4, p_{W}(2)=1 / 8$. Then $p_{W}(1)^{2}-$ $p_{W}(0) p_{W}(2)=-1 / 64$ and so $W$ is not log-concave. However, $p_{W}(1) / p_{W}(0)=2 / 5$ and $p_{W}(2) / p_{W}(1)=1 / 2$ and so, by Lemma 4.3 below, $W+\operatorname{Geom}(p)$ is log-concave for any $p \geq 1 / 2$.

Definition 4.1. For each random variable $V$, define the set

$$
\mathcal{C}_{V}=\{W \text { independent of } V: V+W \text { is log-concave }\} .
$$

Define a partial order on random variables by $V_{1} \preceq V_{2}$ if and only if $\mathcal{C}_{V_{1}} \subseteq \mathcal{C}_{V_{2}}$. Write $L C$ for the set of log-concave variables.

We first give some simple properties of the sets $\mathcal{C}_{V}$, before going on to consider properties of the $\operatorname{sets} \mathcal{C}_{\mathrm{Geom}(p)}$.

Proposition 4.2. For the sets $\mathcal{C}_{V}$ defined above:

(1) If $W \in \mathcal{C}_{V}$ then $V \in \mathcal{C}_{W}$.

(2) For each $V, \mathcal{C}_{V}$ is a closed set.

(3) The $\mathcal{C}_{V}$ are partial order ideals with respect to $\preceq$. That is, given $W_{1} \preceq W_{2}$, if $W_{1} \in \mathcal{C}_{V}$ then $W_{2} \in \mathcal{C}_{V}$.

(4) $\bigcap_{V \in L C} \mathcal{C}_{V}=L C$.

(5) If $V_{2}=V_{1}+U$, where $V_{1}$ and $U$ are independent and $U$ is log-concave, then $V_{1} \preceq V_{2}$.

Proof. The first result is trivial. For each $i$, the set of $W$ with $p_{V+W}(i)^{2} \geq p_{V+W}(i-1) p_{V+W}(i+1)$ is closed, since it can be expressed as the inverse image of the closed set $[0, \infty)$ under a continuous map which depends only on $p_{W}(0), \ldots, p_{W}(i+1)$. This means that $\mathcal{C}_{V}$ is a countable intersection of closed sets and so Part 2 follows.

Part 3 is an application of Part 1 , since

$$
W_{1} \in \mathcal{C}_{V} \Rightarrow V \in \mathcal{C}_{W_{1}} \Rightarrow V \in \mathcal{C}_{W_{2}} \Rightarrow W_{2} \in C_{V}
$$

To prove Part 4, note that Theorem 1.4 entails that for all $V \in L C, L C \subseteq \mathcal{C}_{V}$, so $L C \subseteq \bigcap_{V \in L C} \mathcal{C}_{V}$. Further, since for $V \equiv 0$ we have $\mathcal{C}_{V}=L C$, this set inclusion must be an equality.

For any $W \in \mathcal{C}_{V_{1}}$, the random variable $V_{1}+W$ is log-concave and so by Theorem $1.4\left(V_{1}+W\right)+U=\left(V_{2}+W\right)$ is log-concave. Hence $W \in \mathcal{C}_{V_{2}}$ and Part 5 follows.

Lemma 4.3. For a random variable $X$ with probability mass function $p_{X}(i)$ :

(1) If $p_{X}(i+1) / p_{X}(i) \leq p$ for all $i \geq 1$, then $X \in \mathcal{C}_{\mathrm{Geom}(p)}$.

(2) If there is a gap in the support of $X$ (that is for some $i, p_{X}(i)=0$, but $p_{X}(i-1)>0$ and $p_{X}(i+1)>0$ ) then $X \notin \mathcal{C}_{\mathrm{Geom}(p)}$ for any $p$.

Proof. Write $q_{p}(i)$ for the probability mass function of $X+\operatorname{Geom}(p)$. Note that since

$$
q_{p}(i)=\sum_{j=0}^{i}(1-p) p^{i-j} p_{X}(j)=(1-p) p_{X}(i)+p q_{p}(i-1)
$$

we have

$$
\begin{aligned}
& q_{p}(i)^{2}-q_{p}(i+1) q_{p}(i-1) \\
& \quad=q_{p}(i)\left\{(1-p) p_{X}(i)+p q_{p}(i-1)\right\}-q_{p}(i-1)\left\{(1-p) p_{X}(i+1)+p q_{p}(i)\right\} \\
& \quad=(1-p)\left\{q_{p}(i) p_{X}(i)-q_{p}(i-1) p_{X}(i+1)\right\} .
\end{aligned}
$$


This means that the sum $X+\operatorname{Geom}(p)$ is log-concave if and only if for all $i \geq 1$,

$$
q_{p}(i) p_{X}(i)-q_{p}(i-1) p_{X}(i+1) \geq 0 .
$$

By (10), we have that $q_{p}(i) \geq p q_{p}(i-1)$ and so if $p_{X}(i+1) / p_{X}(i) \leq p$ for all $i \geq 1$, then

$$
\frac{p_{X}(i+1)}{p_{X}(i)} \leq p \leq \frac{q_{p}(i)}{q_{p}(i-1)},
$$

and so $X+\operatorname{Geom}(p)$ is log-concave.

If $p_{X}(i-1)>0$ then $q_{p}(i-1)>0$. Hence, if $p_{X}(i)=0$ and $p_{X}(i+1)>0$ then the inequality (12) fails to hold for this particular $i$, so $X+\operatorname{Geom}(p)$ is not log-concave.

We now use arguments suggested by the proof of Theorem 1.5 to show that the property that $X+\operatorname{Geom}(p)$ is log-concave holds monotonically in $p$. The key, as in Equation (5), is to expand $q_{p_{2}}(i)^{2}-q_{p_{2}}(i-1) q_{p_{2}}(i+1)$ as a sum of terms of the form $q_{p_{1}}(j) q_{p_{1}}(k) a_{j, k}^{(i)}$. This is achieved in Equation (14).

Theorem 4.4. The partial order $\preceq$ induces a total order on the set of geometric random variables, that is:

$$
\operatorname{Geom}\left(p_{1}\right) \preceq \operatorname{Geom}\left(p_{2}\right) \text { if and only if } p_{1} \leq p_{2} .
$$

Proof. With the notation of Lemma 4.3, if $p_{1} \leq p_{2}$, there exist positive constants $b_{r}$, where $b_{0}=\left(1-p_{2}\right) /\left(1-p_{1}\right)$, $b_{r}=\left(\left(p_{2}-p_{1}\right)\left(1-p_{2}\right) /\left(1-p_{1}\right)\right) p_{2}^{r-1}$, such that

$$
q_{p_{2}}(i)=\sum_{r=0}^{i} b_{r} q_{p_{1}}(i-r)
$$

This follows using a standard coupling argument, or since

$$
\begin{aligned}
\frac{\left(p_{2}-p_{1}\right)\left(1-p_{2}\right)}{\left(1-p_{1}\right)} \sum_{r=0}^{i} p_{2}^{r-1} q_{p_{1}}(i-r) & =\frac{\left(p_{2}-p_{1}\right)\left(1-p_{2}\right)}{p_{2}} \sum_{j=0}^{i} q(j) \sum_{j=0}^{i-j} p_{2}^{r} p_{1}^{i-r-j} \\
& =\frac{\left(1-p_{2}\right)}{p_{2}} \sum_{j=0}^{i} q(j)\left(p_{2}^{i-j+1}-p_{1}^{i-j+1}\right) \\
& =q_{p_{2}}(i)-\frac{p_{1}\left(1-p_{2}\right)}{p_{2}\left(1-p_{1}\right)} q_{p_{1}}(i) .
\end{aligned}
$$

Now, we can combine equations (10), (11) and (13) to obtain:

$$
\begin{aligned}
q_{p_{2}}(i)^{2}-q_{p_{2}}(i+1) q_{p_{2}}(i-1)= & \left(1-p_{2}\right)\left\{q_{p_{2}}(i) p_{X}(i)-q_{p_{2}}(i-1) p_{X}(i+1)\right\} \\
= & \frac{\left(1-p_{2}\right)}{\left(1-p_{1}\right)} \sum_{r=0}^{i} b_{r}\left\{q_{p_{1}}(i-r)\left(q_{p_{1}}(i)-p_{1} q_{p_{1}}(i-1)\right)\right. \\
& \left.-q_{p_{1}}(i-r-1)\left(q_{p_{1}}(i+1)-p_{1} q_{p_{1}}(i)\right)\right\} .
\end{aligned}
$$

Now, each term in curly brackets is positive, since $q_{p_{1}}(i)-p_{1} q_{p_{1}}(i-1) \geq 0$, so by log-concavity of $q_{p_{1}}$, for all $r \geq 0$ :

and so $q_{p_{2}}(i)^{2}-q_{p_{2}}(i+1) q_{p_{2}}(i-1) \geq 0$.

$$
\frac{q_{p_{1}}(i-r)}{q_{p_{1}}(i-r-1)} \geq \ldots \geq \frac{q_{p_{1}}(i)}{q_{p_{1}}(i-1)} \geq \frac{q_{p_{1}}(i+1)-p_{1} q_{p_{1}}(i)}{q_{p_{1}}(i)-p_{1} q_{p_{1}}(i-1)},
$$


Acknowledgements. We are grateful to anonymous referees whose comments led to considerable improvements in the presentation of this paper.

\section{REFERENCES}

[1] T. Bergstrom and M. Bagnoli, Log-concave probability and its applications. Econom. Theory 26 (2005) $445-469$.

[2] B. Biais, D. Martimort and J.-C. Rochet, Competing mechanisms in a common value environment. Econometrica 68 (2000) 799-837.

[3] M. Bóna and R. Ehrenborg, A combinatorial proof of the log-concavity of the numbers of permutations with $k$ runs. J. Combin. Theory Ser. A 90 (2000) 293-303.

[4] F. Brenti, Unimodal, log-concave and Pólya frequency sequences in combinatorics. Mem. Amer. Math. Soc. 81 (1989) viii+106.

[5] F. Brenti, Expansions of chromatic polynomials and log-concavity. Trans. Amer. Math. Soc. 332 (1992) $729-756$.

[6] F. Brenti, Log-concave and unimodal sequences in algebra, combinatorics, and geometry: an update in Jerusalem combinatorics '93, Amer. Math. Soc., Providence, RI, Contemp. Math. 178 (1994) 71-89.

[7] H. Davenport and G. Pólya, On the product of two power series. Canadian J. Math. 1 (1949) 1-5.

[8] V. Gasharov, On the Neggers-Stanley conjecture and the Eulerian polynomials. J. Combin. Theory Ser. A 82 (1998) $134-146$.

[9] S.G. Hoggar, Chromatic polynomials and logarithmic concavity. J. Combin. Theory Ser. B 16 (1974) $248-254$.

[10] K. Joag-Dev and F. Proschan, Negative association of random variables with applications. Ann. Statist. 11 (1983) $286-295$.

[11] E.H. Lieb, Concavity properties and a generating function for Stirling numbers. J. Combin. Theory $\mathbf{5}$ (1968) $203-206$.

[12] E.J. Miravete, Preserving log-concavity under convolution: Comment. Econometrica 70 (2002) $1253-1254$.

[13] C.P. Niculescu, A new look at Newton's inequalities. JIPAM. J. Inequal. Pure Appl. Math. 1 (2000) Issue 2, Article 17; see also http://jipam.vu.edu.au/.

[14] R.C. Read, An introduction to chromatic polynomials. J. Combin. Theory 4 (1968) 52-71.

[15] B.E. Sagan, Inductive and injective proofs of log concavity results. Discrete Math. 68 (1988) $281-292$.

[16] B.E. Sagan, Inductive proofs of $q$-log concavity. Discrete Math. 99 (1992) 289-306.

[17] R.P. Stanley, Log-concave and unimodal sequences in algebra, combinatorics, and geometry, in Graph theory and its applications: East and West (Jinan, 1986), Ann. New York Acad. Sci., New York Acad. Sci., New York 576 (1989) $500-535$.

[18] Y. Wang, Linear transformations preserving log-concavity. Linear Algebra Appl. 359 (2003) $161-167$.

[19] Y. Wang and Y.-N. Yeh, Log-concavity and LC-positivity. Available at arXiv:math. C0/0504164 (2005). To appear in J. Combin. Theory $\operatorname{Ser} A$.

[20] Y. Wang and Y.-N. Yeh, Polynomials with real zeros and Pólya frequency sequences. J. Combin. Theory Ser. A 109 (2005) $63-74$.

[21] D.J.A. Welsh, Matroid theory, L.M.S. Monographs, No. 8. Academic Press, London (1976).

[22] H.S. Wilf, Generatingfunctionology. Academic Press Inc., Boston, MA, second edition (1994). 